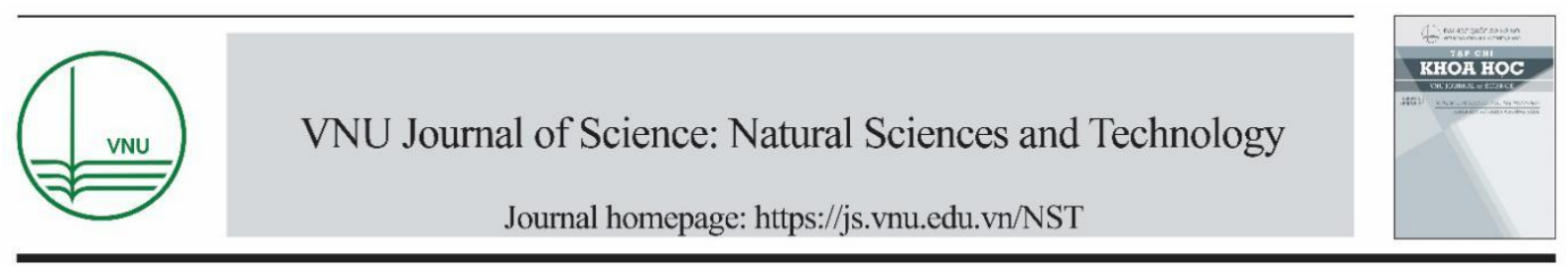

Original Article

\title{
Synthesis and In vitro Cytotocxic Evaluation of New Quinazolinone Derivatives
}

\author{
Tran Dang Thinh, Tran Khac Vu* \\ School of Chemical Engineering, Hanoi University of Science and Technology \\ No1 Dai Co Viet, Hai Ba Trung, Hanoi, Vietnam
}

Received 23 February 2020

Revised 22 July 2020; Accepted 6 August 2020

\begin{abstract}
The paper presents a simple and efficient synthesis of a series of new quinazolinone derivatives 8a-h. First, the reaction of 5-hydroxyanthranilic acid (6) with acetic anhydride at reflux temperature for $2 \mathrm{~h}$ gave the intermediate 7 in high yield. This intermediate was then reacted with amines in acetic acid at reflux temperature $14 \mathrm{~h}$ afforded new quinazolinone derivatives 8a-h in 77$92 \%$. Synthesized compounds were structurally confirmed using spectroscopic methods: ${ }^{1} \mathrm{H}$, ${ }^{13} \mathrm{CNMR}$ and mass spectra. The bioassay result using three cancer cell lines including SKLU-1 (lung cancer), MCF-7 (breast cancer) and HepG-2 (liver cancer) showed that only compound 8h exhibited significant cytotoxic effect against cancer cell lines tested with $\mathrm{IC}_{50}$ values of $23.09,27.75$ and 30.19 $\mu \mathrm{g} / \mathrm{mL}$, respectively.
\end{abstract}

Keywords: Quinazolinone, cytotoxicity, cancer, base, in vitro, derivative.

\footnotetext{
* Corresponding author.

Email address:vu.trankhac@hust.edu.vn

https://doi.org/10.25073/2588-1140/vnunst.5004
} 


\title{
Tổng hợp và đánh giá tác dụng độc tế bào các dẫn xuất mới quinazolinone
}

\author{
Trần Đăng Thịnh, Trần Khắc Vũ* \\ Truòng Đại học Bách khoa Hà Nội, Số 1 - Đại Cồ Việt, Hai Bà Trung, Hà Nội, Việt Nam \\ Nhận ngày 23 tháng 2 năm 2020 \\ Chỉnh sửa ngày 22 tháng 7 năm 2020; Chấp nhận đăng ngày 6 tháng 8 năm 2020
}

\begin{abstract}
Tóm tắt: Bài báo trình bày tổng hợp đơn giản và hiệu quả các dẫn xuất mới của quinazolinone 8ah. Trước tiên, phản ứng của axit 5-hydroxyanthranilic (6) với anhyđrit axetic ở nhiệt độ hồi lưu trong 2 giờ cho chất trung gian 7 với hiệu suất cao. Hợp chất trung gian này sau đó phản ứng với các amin trong axit axetic ở nhiệt độ hồi lưu trong 14 giờ cho một loạt các dẫn xuất mới quinazolinone 8a-h (77-92\%). Các hợp chất tổng hợp được khẳng định cấu trúc sử dụng các phương pháp phổ ${ }^{1} \mathrm{H}$, ${ }^{13} \mathrm{CNMR}$ và phổ khối. Kết quả đánh giá hoạt tính gây độc tế bào với ba dòng tế bào ung thư người: SKLU-1 (ung thư phổi), MCF-7 (ung thư vú) và HepG-2 (ung thư gan) cho thấy chỉ có hợp chất $8 \mathrm{~h}$ thể hiện hoạt tính gây độc ý nghĩa với các dòng tế bào đã thử với giá trị $\mathrm{IC}_{50}$ tương ứng: 23,09; 27,75 và $30,19 \mu \mathrm{g} / \mathrm{mL}$.
\end{abstract}

Tù khóa: Quinazolinone, cytotoxicity, cancer, base, in vitro, derivative.

\section{Mở đầu}

Ung thư hiện đang là mối quan tâm sức khỏe cả ở những nước đang và đã phát triển. Mặc dù có nhiều tiến bộ vượt bậc trong những thập kỷ vừa qua, số ca tử vong vì ung thư vẫn không ngừng tăng. Chỉ tính riêng ở Mỹ, một nước có nền khoa học tiên tiến bậc nhất thế giới, năm 2017 có tới 1,6 triệu trường hợp mắc mới và hơn 600.000 trường hợp tử vong $[1,2]$. Một trong những nguyên nhân chính là sự kháng thuốc và các tác dụng phụ bất lợi của hóa trị liệu [3]. Để phát triển các tác nhân trị ung thư mới hiệu quả hơn, việc nghiên cứu các tác nhân mới với cơ chế mới là một việc thực sự cấp bách. Trong những năm gần đây, sự quan tâm ngày càng tăng đối với nghiên cứu phát triển thuốc ung thư từ các hợp chất hữu cơ dị vòng [4-6]. Đáng chú ý, trong số các cấu trúc dị vòng, quinazolinone đóng một vai trò rất quan trọng do các tương tác tiềm năng $\pi$ $\pi$ và liên kết hydro với các axit amin của thụ thể $\alpha 1$ và $A I I[7]$ và quinazolinone được coi là khung cơ bản quyết định hoạt tính sinh học trong rất

\footnotetext{
* Tác giả liên hệ.

Địa chỉ email: vu.trankhac@ hust.edu.vn
}

https://doi.org/10.25073/2588-1140/vnunst.5004 nhiều loại thuốc. Các nghiên cứu cho thấy một số dẫn xuất quinazolinone (1-5) đã được báo cáo thể hiện các hoạt tính dược lý khác nhau, bao gồm chống ung thư [8-11], chống oxy hóa [12], chống vi rút [13], chống co giật [14], chống viêm [15], chống nhiê̂m trùng [16], chống HIV [17], v.v. Hơn nữa, quinazolinone và các dẫn xuất của chúng cũng được báo cáo cho thấy một số ưu điểm so với các tác nhân đang được sử dụng lâm sàng [18] và có liên quan chặt chẽ với các liệu pháp chống ung thư $[19,20]$. Một số dẫn xuất quinazolinone đã được chứng minh trong điều trị bệnh bạch cầu ở người so với các tác nhân thông thường và cho thấy tác dụng đáng kể của chúng đối với các dòng tế bào ung thư vú [21-24]. Vì vậy, để tiếp tục tìm kiếm các cấu trúc mới có tác dụng kháng ung thư, chúng tôi tiếp tục tập trung vào việc tổng hợp các dẫn xuất mới quinazolinone và đánh giá tác dụng gây độc của chúng trên một số dòng tế bào ung thư. Bài báo này trình bày kết quả tổng hợp và đánh giá tác dụng sinh học của các dẫn xuất mới quinazolinone. 
<smiles>Cc1ccc(-c2nc3ccc(Cl)cc3c(=O)n2NC(=O)CNN)cc1</smiles>

1<smiles>Cc1ccc(-c2nc3ccc(Cl)cc3c(=O)n2/N=C/c2ccccc2)cc1</smiles>

2<smiles>O=c1c2cc(Cl)ccc2nc(-c2ccccc2)n1N=Cc1ccc(Cl)cc1</smiles><smiles>Cc1nc2ccccc2c(=O)n1Nc1cnc(N)s1</smiles><smiles>Cc1nc2ccccc2c(=O)n1NC(=O)/C(Cl)=C/c1ccccc1</smiles>

Hình 1. Một số dẫn xuất quinazolinone có tác dụng kháng ung thư [8].

\section{Thực nghiệm}

Các tác nhân phản ứng được mua từ các hãng Aldrich hay Merck. Dung môi được mua từ Trung Quốc, được cất lại trước khi dùng. Điểm chảy được đo trên máy Electrothermal IA 9200 Shimadzu. Phổ ESI-MS được đo trên máy FTICR MS Varian và phổ ${ }^{1} \mathrm{H}$ NMR và ${ }^{13} \mathrm{C}$ NMR được đo trên máy Bruker AVANCE $500 \mathrm{MHz}$ tại Viện Hóa học, Viện Hàn lâm Khoa học và Công nghệ Việt Nam sử dụng dung môi đo: $\mathrm{CD}_{3} \mathrm{OD}$ và $\mathrm{DMSO}-\mathrm{d} 6$. Độ chuyển dịch hóa học

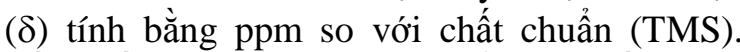
Hằng số tương tác $(\mathrm{J})$ được biểu diễn bằng $\mathrm{Hz}$. Tiến trình phản ứng được theo dõi bởi sắc ký lớp mỏng (TLC) sử dụng bản nhôm tráng sẵn (Merck 60 F254). Bản mỏng hiện màu bằng thuốc thử vanillin trong axit sunfuric. Sắc ký cột sử dụng silica gel cỡ hạt 40-230 mesh. Đánh giá hoạt tính gây độc tế bào được thực hiện tại viện Công nghệ sinh học, Viện Hàn lâm Khoa học và Công nghệ Việt Nam.

Tổng hơp 6-hydroxy-2methyl-4H-benzo[d] [1,3] oxazin-4-one (7)

Một hỗn hợp của axit 5-hydroxyanthranilic (6) $(5,0 \mathrm{~g} ; 32,67 \mathrm{mmol})$ trong anhyđrit axetic (15 $\mathrm{ml}$ ) được đun hồi lưu trong 2 giờ. Hỗn hợp phản ứng sau đó được đổ ra nước đá. Tủa tạo thành được lọc, rửa với nước cất và làm khô trong chân không cho sản phẩm 7 (5,03 g; 87\%) là chất rắn màu nâu nhạt được xử dụng luôn cho bước tiếp theo [25].

\section{Quy trình chung tổng hợp chất $8 a-h$}

Một hỗn hợp của 7 (1,0 g; 5,64 mmol) và các amin $(3 \mathrm{eq})$, axit axetic $(10 \mathrm{~mL})$ được đun hồi lưu trong 14 giờ. Tiến trình phản ứng được kiểm tra bởi TLC ( $n$-hexan:etyl axetat $=1: 1)$. Hỗn hợp phản ứng sau đó được trung hòa bằng $50 \%$ $\mathrm{NaHCO}_{3}$ tới $\mathrm{pH}=7$, và chiết với $\mathrm{CH}_{2} \mathrm{Cl}_{2}(3 \times 20$ $\mathrm{mL})$. Pha hữu cơ được tách ra, làm khan bằng $\mathrm{Na}_{2} \mathrm{SO}_{4}$ và bay hơi dưới áp suất giảm cho các cặn tương ứng. Các cặn này sau đó được tiến hành sắc ký cột silica gel sử dụng hệ dung môi rửa dải $n$-hexan / etyl axetat phù hợp cho các chất 8a-h.

\section{3-Cyclopropyl-6-hydroxy-2-methylquina} zolin -4(3H)-one $(8 a)$ : Chất rắn màu vàng; hiệu suất: $88 \%$; đnc: $243-244{ }^{\circ} \mathrm{C} ; R_{f}=0,57$ ( $n$-hexan: etyl axetat $=1: 1) ;{ }^{1} \mathrm{H}$ NMR $(500 \mathrm{MHz}$, DMSO$d 6, \delta(\mathrm{ppm})): 7,87(\mathrm{~d}, J=3,0 \mathrm{~Hz}, 1 \mathrm{H}) ; 7,51(\mathrm{~d}, J$ $=9,0 \mathrm{~Hz}, 1 \mathrm{H}) ; 7,29-7,27(\mathrm{dd}, J=3,0 \mathrm{~Hz}, 9,0 \mathrm{~Hz}$, $1 \mathrm{H}) ; 2.96(\mathrm{~m}, 1 \mathrm{H}) ; 2,71\left(\mathrm{~s}, 3 \mathrm{H}, \mathrm{CH}_{3}\right) ; 1,33(\mathrm{~m}$, $2 \mathrm{H}) ; 0,95(\mathrm{~m}, 2 \mathrm{H}) .{ }^{13} \mathrm{C}$ NMR (125 MHz, DMSOd6, $\delta$ (ppm)): 163,4; 155,3; 153,8; 141,2; 128,2; 124,$0 ; 121,8,110,2 ; 27,8 ; 23,2 ; 10,4$. ESI-MS $\mathrm{m} / \mathrm{z}: 217,4[\mathrm{M}+\mathrm{H}]^{+}$.

6-Hydroxy-3-(2-methoxyphenyl)-2-methyl quinazolin-4(3H)-one $(8 b)$ : Chất rắn màu trắng; hiệu suất: $88 \%$; đnc: $156-157{ }^{\circ} \mathrm{C} ; R_{f}=0,50(n$ hexan: etyl axetat $=1: 1)$; ${ }^{1} \mathrm{H}$ NMR $(500 \mathrm{MHz}$, DMSO-d6, $\delta$ (ppm)): 10,31 (brs, 1H, OH); 7,55 $(\mathrm{m}, 2 \mathrm{H}) ; 7,38(\mathrm{~d}, J=2,5 \mathrm{~Hz}, 1 \mathrm{H}) ; 7,35(\mathrm{dd}, J=$ $1,5 \mathrm{~Hz}, 7,5 \mathrm{~Hz}, 1 \mathrm{H}) ; 7,29$ (dd, $J=2,5 \mathrm{~Hz}, 8,50$ $\mathrm{Hz}, 1 \mathrm{H}) ; 7,25(\mathrm{~d}, J=8,50 \mathrm{~Hz}, 1 \mathrm{H}) ; 7,11(\mathrm{t}, J=$ 
7,5 Hz, 1H); 3,76 (s, 3H); 2,04 (s, 3H). ${ }^{13} \mathrm{C} \mathrm{NMR}$ (125 MHz, DMSO-d6, $\delta$ (ppm)): 160,6; 155,8; 154,$2 ; 151,3 ; 140,6 ; 130,6 ; 129,6 ; 128,3 ; 126,2$; 123,$9 ; 121,2 ; 120,9 ; 112,4 ; 109,2 ; 55,7 ; 22,7$. ESI-MS m/z: 283,2 [M+H] ${ }^{+}$.

6-Hydroxy-3-(3-methoxyphenyl)-2-methyl quinazolin-4(3H)-one $(8 c)$ : Chất rắn màu trắng; hiệu suất: $92 \% ; R_{f}=0,49$ ( $n$-hexan: etyl axetat $=$ $1: 1)$; ${ }^{1} \mathrm{H}$ NMR $\left(500 \mathrm{MHz}, \mathrm{CD}_{3} \mathrm{OD}, \delta(\mathrm{ppm})\right)$ : $7,59(\mathrm{~d}, J=9,0 \mathrm{~Hz}, 1 \mathrm{H}) ; 7,52-7,49(\mathrm{~m}, 2 \mathrm{H}) ; 7,35$ (dd, $J=3,0 \mathrm{~Hz}, 9,0 \mathrm{~Hz}, 1 \mathrm{H}) ; 7,13(\mathrm{dd}, J=6,0$ $\mathrm{Hz}, 8,5 \mathrm{~Hz}, 1 \mathrm{H}) ; 6,98$ (t, $J=7,0 \mathrm{~Hz}, 1 \mathrm{H}) ; 6,94$ $(\mathrm{d}, J=8,5 \mathrm{~Hz}, 1 \mathrm{H}) ; 3,87\left(\mathrm{~s}, 3 \mathrm{H}, \mathrm{OCH}_{3}\right) ; 2,25$ (s, $\left.3 \mathrm{H}, \mathrm{CH}_{3}\right) .{ }^{13} \mathrm{C}$ NMR $\left(125 \mathrm{MHz}, \mathrm{CD}_{3} \mathrm{OD}, \delta\right.$ (ppm)): 162,7; 162,5; 157,9; 153,5, 141,9; 140,2; 131,$7 ; 128,9 ; 125,6 ; 122,7 ; 121,4 ; 116,3 ; 115,0$; $110,6 ; 56,1 ; 23,5$. ESI-MS m/z: 283,2 [M+H] $]^{+}$.

6-Hydroxy-3-(4-methoxyphenyl)-2-methyl quinazolin-4(3H)-one $(8 d)$ : Chất rắn màu trắng (chất cũ) [22]; hiệu suất: $79 \%$; đnc: $263-264^{\circ} \mathrm{C}$; $R_{f}=0,45$ ( $n$-hexan: etyl axetat $\left.=1: 1\right) ;{ }^{1} \mathrm{H}$ NMR $\left(500 \mathrm{MHz}, \mathrm{CD}_{3} \mathrm{OD}, \delta(\mathrm{ppm})\right): 7,58(\mathrm{~d}, J=9,0$ $\mathrm{Hz}, 1 \mathrm{H}) ; 7,51(\mathrm{~d}, J=2,50 \mathrm{~Hz}, 1 \mathrm{H}) ; 7,4(\mathrm{dd}, J=$ $2,50 \mathrm{~Hz}, 9,0 \mathrm{~Hz}, 1 \mathrm{H}) ; 7,28(\mathrm{~d}, J=8,50 \mathrm{~Hz}, 2 \mathrm{H})$; $7,14(\mathrm{~d}, J=8,50 \mathrm{~Hz}, 2 \mathrm{H}) ; 3,90(\mathrm{~s}, 3 \mathrm{H}) ; 2,22$ (s, $3 \mathrm{H}) .{ }^{13} \mathrm{C}$ NMR $\left(125 \mathrm{MHz}, \mathrm{CD}_{3} \mathrm{OD}, \delta(\mathrm{ppm})\right.$ ): 164,$1 ; 161,7 ; 157,9 ; 154,1,141,9 ; 131,6 ; 130,4$; 128,$8 ; 125,6 ; 122,7 ; 116,3 ; 110,6 ; 56,1 ; 23,7$. ESI-MS m/z: 283,2 [M+H] ${ }^{+}$.

3-(4-Fluorophenyl)-6-hydroxy-2-methylqui nazolin-4(3H)-one $(8 \mathrm{e})$ : Chất rắn màu vàng sáng; $177-178{ }^{\circ} \mathrm{C}$; hiệu suất: $82 \% ; R_{f}=0,51(n$ hexan:etyl axetat $=1: 1) ;{ }^{1} \mathrm{H}$ NMR $(500 \mathrm{MHz}$, DMSO-d6, $\delta(\mathrm{ppm})): 7,57(\mathrm{~d}, J=9,0 \mathrm{~Hz}, 1 \mathrm{H}, \mathrm{H}-$ 8); 7,43 (s, $J=3,0 \mathrm{~Hz}, 1 \mathrm{H}, \mathrm{H}-5) ; 7,42-7,41$ (dd, $J=3,0 \mathrm{~Hz}, 9,0 \mathrm{~Hz}, 2 \mathrm{H}) ; 7,36-7,32(\mathrm{~m}, 3 \mathrm{H}) ; 4,83$ (s, 2H); 2,21 (s, 3H). ${ }^{13} \mathrm{C}$ NMR $(125 \mathrm{MHz}$, DMSO-d6, $\delta$ (ppm)): 165,3; 163,8; 163,3; 157,9; 141,$9 ; 135,2 ; 131,7 ; 128,9 ; 125,6 ; 122,6 ; 117,8$; 117,$7 ; 110,6 ; 23,7$. ESI-MS m/z: 271,5 [M+H] ${ }^{+}$.

3-(2-Chlorophenyl)-6-hydroxy-2-methylqui nazolin-4(3H)-one $(8 f)$ : Chất rắn màu trắng; hiệu suất: $81 \%$; đnc: $299-300{ }^{\circ} \mathrm{C} ; R_{f}=0,47(n$ hexan: etyl axetat $=1: 1)$; ${ }^{1} \mathrm{H}$ NMR $(500 \mathrm{MHz}$, DMSO-d6, $\delta(\mathrm{ppm}))$ : 7,73-7,71 (m, 1H); 7,59 $(\mathrm{m}, 3 \mathrm{H}) ; 7,55-7,52(\mathrm{~m}, 2 \mathrm{H}) ; 7,38(\mathrm{dd}, J=2,5 \mathrm{~Hz}$,
$8,5 \mathrm{~Hz}, 1 \mathrm{H}) ; 2,16$ (s, 3H, $\left.\mathrm{CH}_{3}\right) \cdot{ }^{13} \mathrm{C}$ NMR $(125$ MHz, DMSO-d6, $\delta$ (ppm)): 163,0; 158,2; 152,7; 141,$9 ; 136,6 ; 133,5 ; 132,4 ; 131,7 ; 129,8 ; 129,1$; 125,$8 ; 122,5 ; 110,6 ; 22,9$. ESI-MS m/z: 287,4 $[\mathrm{M}+\mathrm{H}]^{+}$.

3-(3-Fluorophenyl)-6-hydroxy-2-methyl quinazolin-4(3H)-one $(8 \mathrm{~g})$ : Chất rắn màu trắng; hiệu suất: $83 \% ; R_{f}=0,54$ ( $n$-hexan:etyl axetat $=$ 1:1); ${ }^{1} \mathrm{H}$ NMR (500 MHz, $\mathrm{CD}_{3} \mathrm{OD}, \delta$ (ppm)): $7,63(\mathrm{~m}, 1 \mathrm{H}) ; 7,57(\mathrm{~d}, J=9,0 \mathrm{~Hz}, 1 \mathrm{H}) ; 7,50(\mathrm{~d}, J$ $=3,0 \mathrm{~Hz}, 1 \mathrm{H}) ; 7,35-7,32(\mathrm{~m}, 2 \mathrm{H}) ; 7,28-7,25(\mathrm{~m}$, 1H); 7,24-7,22 (m, 1H); 3,25 (s, 3H). ${ }^{13} \mathrm{C} \mathrm{NMR}$ $\left(125 \mathrm{MHz}, \mathrm{CD}_{3} \mathrm{OD}, \delta\right.$ (ppm)): 165,6; 163,7; 158,$0 ; 153,0 ; 141,9 ; 140,7 ; 132,5 ; 129,0 ; 125,7$; 125,$6 ; 122,6 ; 117,5 ; 117,1 ; 110,6 ; 23,6$. ESI-MS $\mathrm{m} / \mathrm{z}: 271,5[\mathrm{M}+\mathrm{H}]^{+}$.

3- (4-Acetylphenyl)-6-hydroxy-2-methylqui nazolin-4(3H)-one (8h): Chất rắn màu trắng; hiệu suất: $77 \%$; đnc: $247-248{ }^{\circ} \mathrm{C} ; R_{f}=0,53(n-$ hexan:etyl axetate $=1: 1) ;{ }^{1} \mathrm{H}$ NMR $(500 \mathrm{MHz}$, DMSO-d6, $\delta(\mathrm{ppm})): 10,03(\mathrm{~s}, 1 \mathrm{H}, \mathrm{OH}) ; 8,13(\mathrm{~d}$, $J=8,5 \mathrm{~Hz}, 2 \mathrm{H}) ; 7,60(\mathrm{~d}, J=8,50 \mathrm{~Hz}, 2 \mathrm{H}) ; 7,55$ $(\mathrm{d}, J=9,0 \mathrm{~Hz}, 1 \mathrm{H}) ; 7,40(\mathrm{~d}, J=3,0 \mathrm{~Hz}, 1 \mathrm{H}) ; 7,30$ (dd, $J=3,0 \mathrm{~Hz}, 9,0 \mathrm{~Hz}, 1 \mathrm{H}) ; 2,65\left(\mathrm{~s}, 3 \mathrm{H}, \mathrm{CH}_{3}\right)$; 2,08 (s, $\left.3 \mathrm{H}, \mathrm{CH}_{3}\right) .{ }^{13} \mathrm{C}$ NMR $(125 \mathrm{MHz}$, DMSOd6, $\delta$ (ppm)): 197,3; 170,3; 160,9; 155,9; 150,2; 142,$1 ; 140,5 ; 136,9 ; 129,4 ; 129,0 ; 128,3 ; 124,0$; 121,$2 ; 109,1 ; 26,8 ; 23,6$. ESI-MS m/z: 295,6 $[\mathrm{M}+\mathrm{H}]$.

\section{Kết quả và thảo luận}

\subsection{Hóa họ}

Các dẫn xuất mới 8a-h được tổng hợp như ở sơ đồ 1. Trước tiên hợp chất axit 5hydroxyanthranilic $(\mathbf{6})$ được phản ứng với lượng dư anhydrit axetic ở nhiệt độ hồi lưu trong 2 giờ cho hợp chất trung gian benzoxazinone 7 với hiệu suất $87 \%$. Hợp chất 7 được tinh chế đơn giản bằng cách đổ hỗn hợ phản ứng vào nước đá. Tủa hình thành được lọc và rửa kỹ với nước cất, hút khô được dùng cho bước tiếp theo.

Về cơ chế phản ứng, trong sự có mặt của dư anhyđrit axetic khan và ở nhiệt độ phản ứng cao, nhóm $\mathrm{NH}_{2}$ của axit 5-hydroxyanthranilic nhanh 
chóng tham gia phản ứng axyl hóa để cho hợp chất trung gian 7a. Trong môi trường axit (axetic sinh ra từ phản ứng) chất trung gian này chuyển cân bằng sang dạng imin liên hợp với nhân thơm bền hơn $\mathbf{7 b}$. Cuối cùng sự loại nước dưới tác dụng của nhiệt độ cho sản phẩm trung gian mong muốn 7. Tuy nhiên sản phẩm trung gian này kém bền, dễ hút ẩm và phản ứng với nước để mở vòng thành hợp chất trung gian nên nó được bảo quản khô và dùng luôn cho bước tiếp theo (Hình 2).<smiles>CC(=O)Nc1ccc(O)cc1C(=O)O</smiles>

Hình 2. Cơ chế hình thành chất 7.

Chất 7 tiếp theo được phản ứng với các amin trong điều kiện hồi lưu của axit axetic cho sản phẩm 8a-h với hiệu suất 77-92\%.

Về cơ chế phản ứng, các amin là các nucleophin mạnh. Chúng tấn công vào trung tâm ái điện tử $(\mathrm{C}=\mathrm{O})$ của chất trung gian 7 , vòng oxazin của 7 bị mở ra thành chất trung gian $\mathbf{7 c}$, 7d, 7e. Trong điều kiện của nhiệt độ cao và axit, sản phẩm trung gian bị loại nước cho sản phẩm quinazolinone 8 (Hình 3).

Tất cả các dẫn xuất $\mathbf{8 a}-\mathbf{h}$ được khẳng định cấu trúc dựa trên các phổ ${ }^{1} \mathrm{H}$ NMR và ${ }^{13} \mathrm{C}$ NMR. Do sự tương tự nhau về cấu trúc, hợp chất $\mathbf{8 a}$ được làm ví dụ chứng minh cấu trúc.<smiles>[R]N=CCCCCC1(C)OC(C)=Nc2ccccc21</smiles>

7<smiles>[R]NCC1(C)OC(C)=Nc2ccccc21</smiles>

7b<smiles>[R][NH+](C)C(=O)c1ccccc1N=C(C)OCCCCC</smiles><smiles>[3H]C</smiles><smiles>[R]n1c(C)nc2ccccc2c1=O</smiles>

8

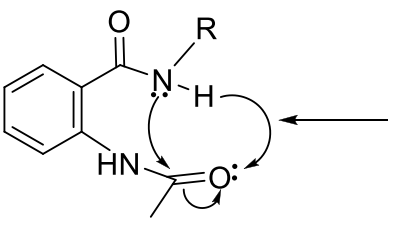

$7 e$<smiles>[R]NC(=O)c1ccccc1N=C(C)O</smiles>

7d

Hình 3. Cơ chế tạo thành hợp chất 8. 
<smiles>Nc1ccc(O)cc1C(=O)O</smiles>

6

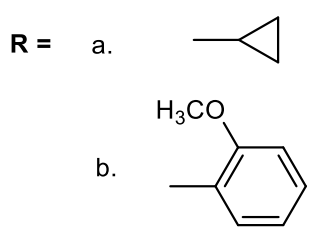<smiles>CC(C)CC1CCCCC1</smiles>

c.<smiles>COc1cccc(C)c1</smiles>

d.<smiles>COc1ccc(C)cc1</smiles>

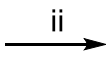<smiles>Cc1ccc(F)cc1</smiles>

f.<smiles>Cc1ccccc1Cl</smiles><smiles>[R]n1c(C)nc2ccc(O)cc2c1=O</smiles>

$8 a-h$

Sơ đồ 1. Điều kiện và tác nhân: (i) $\left(\mathrm{CH}_{3} \mathrm{CO}\right)_{2} \mathrm{O}$, hồi lưu, 2 giờ; (ii) axit axetic, amin, hồi lưu, 14 giờ, 77-92\%.

\subsection{Thư nghiệm hoạt tính sinh học}

Tất cả các hợp chất đích 8a-h được đánh giá độc tính tế bào in vitro. Ba dòng tế bào ung thư ở người bao gồm SKLU-1 (ung thư phổi), MCF7 (ung thư vú) và HepG2 (ung thư gan) được chọn để sàng lọc tác dụng ức chế của chúng bằng phương pháp SRB [26].

Bảng 1 . Hoạt tính gây độc tế bào in vitro các chất $\mathbf{8 a - h}$

\begin{tabular}{|c|c|c|c|c|c|}
\hline \multirow[t]{2}{*}{ TT } & \multirow[t]{2}{*}{ Chất } & \multirow[t]{2}{*}{$\mathbf{R}$} & \multicolumn{3}{|c|}{$\mathrm{IC}_{50}(\mu \mathrm{g} / \mathrm{mL})$} \\
\hline & & & SK-LU-1 & MCF-7 & HepG2 \\
\hline 1 & $8 \mathbf{a}$ & Cycloropyl & $>100$ & $>100$ & $>100$ \\
\hline 2 & $8 \mathbf{b}$ & 2-Methoxyphenyl & $>100$ & $>100$ & $>100$ \\
\hline 3 & $8 c$ & 3-Methoxyphenyl & $>100$ & $>100$ & $>100$ \\
\hline 4 & 8d & 4-Methoxyphenyl & $>100$ & $>100$ & $>100$ \\
\hline 5 & $8 e$ & 4-Fluorophenyl & $>100$ & $>100$ & $>100$ \\
\hline 6 & $8 f$ & 2-Chlorophenyl & $>100$ & $>100$ & $>100$ \\
\hline 7 & $8 g$ & 3-Fluorophenyl & $>100$ & $>100$ & $>100$ \\
\hline 8 & $8 \mathrm{~h}$ & 4-Acetophenyl & $23.09 \pm 2.07$ & $27.75 \pm 1.94$ & $30.19 \pm 0.02$ \\
\hline & Ellipticine & & 0.43 & 0.43 & 0.40 \\
\hline
\end{tabular}

${ }^{\mathrm{a} N o ̂ ̀ n g ~ đ o ̣ ̂ ~}(\mu \mathrm{g} / \mathrm{mL})$ ức chế $50 \%$ tế bào, được tính bằng kết quả trung bình từ 3 thí nghiệm với độ lệch chuẩn

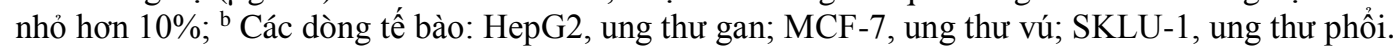

Kết quả hoạt tính (Bảng 1) cho thấy các dẫn xuất quiniazolinone 8a-h không thể hiện hoạt tính đối với ba dòng tế bào ung thư được thử nghiệm ngoại trừ hợp chất $\mathbf{8 h}$ thể hiện hoạt tính gây độc tế bào với các giá trị $\mathrm{IC}_{50}$ lần lượt là $23,09,27,75$ và $30,19 \mu \mathrm{g} / \mathrm{mL}$.

\section{Kết luận}

Chúng tôi đã tổng hợp một loạt các dẫn xuất quinazolinone mới $\mathbf{8} \mathbf{a}-\mathbf{h}$ qua quy trình đơn giản, hiệu suất cao. Cấu trúc của tất cả các hợp chất tổng hợp đã được xác định dựa trên các phổ ${ }^{1} \mathrm{H},{ }^{13} \mathrm{C}$ NMR và MS. Mặc dù kết quả thử nghiệm sinh học cho thấy hầu hết các hợp chất đích không có tác dụng gây độc tế bào so với ellipticine, hợp chất $\mathbf{8 h}$ thể hiện hoạt tính gây độc tế bào ở mức trung bình với các giá trị $\mathrm{IC}_{50}$ là $23,09,27,75$ và $30,19 \mu \mathrm{g} / \mathrm{mL}$, tuy vậy các kết quả này có thể là tài liệu tham khảo hữu ích cho các nghiên cứu tiếp theo về các hợp chất khung quinazolinone. 


\section{Lời cảm ơn}

Công trình này được hoàn thành với sự tài trợ kinh phí từ đề tài Quỹ khoa học và Công nghệ Quốc gia Việt Nam (NAFOSTED), mã số 104.01-2017.05.

\section{Tài liệu tham khảo}

[1] R.L. Siegel, K.D. Miller, Jemal. A, Cancer Statistics, CA Cancer J Clin, 67 (1) (2017)7-30. https://doi.org/10.3322/caac.21395.

[2] R.A. Smith, K.S. Andrews, D. Brooks, S.A. Fedewa, D.M. Baptiste, D.S.O.W. Brawley, R.C. Wender, Cancer Screening in the United States, 2018, A Review of Current American Cancer Society Guidelines and Current Issues in Cancer Screening, CA Cancer J Clin (68) (2018)297-316. https://doi.org/10.3322/caac.21446.

[3] I.K. Mellinghoff, C.L. Sawyers, The emergence of resistance to targeted cancer therapeutics. Pharmacogenomics (3) (2002) 603-623. https:// doi.org/10.1517/14622416.3.5.603.

[4] M.E. Welsch, S.A. Snyder, B.R. Stockwell, Privileged scaffolds for library design and drug discovery, Curr Opin Chem Biol (14) (2010) 1-15. https://doi.org/10.1016/j.cbpa.2010.02.018.

[5] M. Asif, Various chemical and biological activities of pyridazinone derivatives, Cent Eur J Exp Biol (5) (2017) 1-19.

[6] A.P. Asif, K. Bahetia, Computer based drug design of various heterocyclic compounds having anticancer activity: a brief review, J Bioinform Genom Proteom (2) (2017) 1-13.

[7] M.R. Yadav, P.P. Naik, H.P. Gandhi, B.S. Chauhan, R. Giridhar, Design and synthesis of 6,7-dimethoxyquinazoline analogs as multitargeted ligands for $\alpha 1$-and aII-receptors antagonism, Bioorg Med Chem Lett (23) (2013) 3959-3966. https://doi.org/10.1016/j.bmcl.2013. 04.054 .

[8] A. Shetha, I. A. Wijdan, Synthesis and characterization of new quinazoline-4(3H)-one Schiff bases, J Chem Pharm Res (5) (2013) 42-45.

[9] A. M. Alanazi, A.-M. Alaa, Abdel-Aziz, I. A. AlSuwaidan, S. G. Abdel-Hamide, T. Z. Shawer, A. S. El-Azab, Design, synthesis and biological evaluation of some novel substituted quinazolines as antitumor agents, Eur. J. Med. Chem (79) (2014) 446-454.https://doi.org/10.1016/j.ejmech. 2014.04.029.

[10] M. N. Noolvi, H. M. Patel, Synthesis, method optimization, anticancer activity of 2,3,7- trisubstituted Quinazoline derivatives and targeting EGFR-tyrosine kinase by rational approach: 1st Cancer Update, Arab. J. Chem (6) (2013) 35-48. https://doi.org/10.1016/j.arabjc. 2010.12.031.

[11] D. H. Fleita, R. M. Mohareb, O. K. Sakka, Antitumor and antileishmanial evaluation of novel heterocycles derived from quinazoline scaffold: a molecular modeling approach, Med. Chem. Res (22) (2013) 2207-2221. https://doi. org.10.1007/s00044-012-0213-9.

[12] H.M. Vagdevi, M.R. Lokesh, B.C. Gowdarshivannanavar, Synthesis and Antioxidant Activity of 3-Substituted Schiff bases of Quinazoline-2,4-diones, Int J Chem Tech Res (4) (2012) 1527-1533.

[13] S.K. Krishnan, S. Ganguly, R. Veerasamy, B. Ran, Synthesis, antiviral and cytotoxic investigation of 2-phenyl-3-substituted quinazolin -4(3H)-ones, Eur Rev Med Pharm Sci (15) (2011) 673-681.

[14] N.B. Patel, V.N. Patel, H.R. Patel, F.M. Shaikh, J. C. Patel, Synthesis and microbial studies of (4oxo-thiazolidinyl) sulfonamides bearing quinazolin-4(3h) ones, Acta Polo Pharm Drug Res (67) (2010) 267-275.

[15] G. Saravanan, P. Pannerselvam, C.R. Prakash, Synthesis, analgesic and anti-inflammatory screening of novel Schiff bases of 3-amino-2methyl quinazolin 4-(3H)-one, Der Pharmacia Lett (2) (2010) 216-226.

[16] O.H. Abid \& A.H. Ahmed, Synthesis and characterization of novel quinazoline derivatives via reaction of isatoic anhydride with schiff's base, Inter J Appl Nat Sci (2) (2013) 11-20.

[17] B. Pati \& S. Banerjee, Quinazolines: an illustrated review, JAdv Pharm Edu Res (3) (2013) 136-151.

[18] S.N. Katrin, Chemotherapy and Dietary Phytochemical Agents, Chem ther Res Prac (3) (2012) 22-27. doi:10.1155/2012/282570.

[19] A.K. Manasa, R.V. Sidhaye, G. Radhika, C.N. Nalini, Synthesis, antioxidant and anticancer activity of quinazoline derivatives, Current Pharma Research (1) (2011) 101-105.

[20] B. Nerkar, A. Saxena, S. Ghone, A.K. Thakeri, In Silico Screening, Synthesis and In Vitro Evaluation of Some Quinazolinone and Pyridine Derivatives as Dihydrofolate Reductase Inhibitors for Anticancer Activity, E-Journal of Chem (6) (2009) 97-102.

[21] A.V. Danilov, Targeted therapy in chronic lymphocytic leukemia: past, present, and future, Clin Ther (35) (2013) 1258-1270. https://doi.org/ 10.1016/j.clinthera.2013.08.004. 
[22] M.F. Ahmed, M. Youns, Synthesis and Biological Evaluation of a Novel Series of 6, 8-Dibromo-4 $(3 \mathrm{H})$ quinazolinone Derivatives as Anticancer Agents, Archiv der Pharmazie (346) (2013) 610 617. https://doi.org/10.1002/ardp.201300158.

[23] D. Kumar, Design, synthesis and cytotoxic evaluation of novel imidazolone fused quinazolinone derivatives, Arabian J. Chem. https://10.1016/j.arabjc.2014.07.001 (2014).

[24] F.L. Faraj, M. Zahedifard, Synthesis, Characterization, and Anticancer Activity of New Quinazoline Derivatives against MCF-7 Cells, Sci. Wld J. 212096; https://doi.rog/10.1155/2014/ 212096 (2014).

[25] A. Kamal, E.V. Bharathi, M.J Ramaiah, D. Dastagiri, J. S. Reddy, A.Viswanath, F. Sultana,
S.N.C.V.L. Pushpavalli, M.P. Bhadra, H.K. Srivastava, G. N. Sastry.; Aarti J.; Subrata Sen.; Surekha Zingde, Quinazolinone linked pyrrolo[2,1-c][1,4]benzodiazepine (PBD) conjugates: Design, synthesis and biological evaluation as potential anticancer agents, Bioorg. Med. Chem 18 (2), (2010) 526-542. https://doi. org/10.1016/j.bmc.2009.12.015.

[26] D.A. Scudiero, R.H. Shoemaker, K.D. Paull, A. Monks, S. Tierney, T.H. Nofziger, M.J. Currens, D. Seniff, M. Boyd, Feasibility of drug screening with panels of human tumor cell lines using a microculture tetrazolium assay, Cancer Res (48) (1988) 4827-4833. 